\title{
Epigallocatechin gallate enhances the motor neuron survival and functional recovery after brachial plexus root avulsion by regulating FIG4
}

\author{
Yinjuan Tang ${ }^{1}$, Jianjun Wang², Shengjie Wan², Lanbing Luo², Yuxia Qiu², Shiyin Jiang ${ }^{2}$, Guihua Yue², Yumei Tang², \\ Weijun Tang ${ }^{3}$ \\ ${ }^{1}$ Department of Basic Medical Sciences, Xiangnan University, Chenzhou, Hunan Province, China, ${ }^{2}$ Department of Clinical Medicine, \\ Xiangnan University, Chenzhou, Hunan Province, China, ${ }^{3}$ Department of Pharmacy, Xiangnan University, Chenzhou, Hunan Province, \\ China
}

\begin{abstract}
The survival of motor neurons (MNs) is the key to recovery of the motor function after brachial plexus root avulsion (BPRA). (-)-epigallocatechin-3-gallate (EGCG) exerts neuroprotective roles in neurons under different pathological conditions. However, the role of EGCG in regulating motor neurons under BPRA remains to be unclear. In the present study, we investigated the functional role of EGCG both in vitro and in vivo. In an in vitro study, we observed that EGCG obviously increased the cell survival rate of MNs and FIG4 protein levels compared with the vehicle control, with a peak level observed at $50 \mu \mathrm{M}$; EGCG can also upregulate FIG4 to reduce the cell death of MNs and increase the neurite outgrowth under oxidative stress; moreover, EGCG can upregulate FIG4 to promote the functional recovery and the survival of $M N$ s in the ventral horn in mice after BPRA. These combined results may lay the foundation for EGCG to be a novel strategy for the treatment of BPRA.
\end{abstract}

Key words: motor neurons, brachial plexus root avulsion, (-)-epigallocatechin-3-gallate (EGCG), neurodegenerative diseases, FIG4.

\section{Introduction}

Brachial plexus injury is a very serious peripheral nerve injury that is common in clinical practice. It is considered to be one of the world's clinical medical treatment problems, and its incidence has been increasing $[10,13]$. Adult brachial plexus injury often occurs in accidental traffic accidents caused by external factors [14], resulting in the loss of the upper limb motor function, and its clinical treatment effect is not optimistic. Not only does it cause great pain to the individual, it brings heavy economic pressure to the family, but also adds a huge burden to the society. Therefore, the effective treatment of brachial plexus avulsion and its mechanism of action are extremely urgent scientific issues.

Brachial plexus root avulsion is a complex type of nerve injury involving the central and peripheral nervous system. It separates the spinal nerve from the spinal cord and causes serious damage to itself and related spinal segments, resulting in the damage to motor neurons and peripheral nerves. Separation of glial cells causes motoneurons in related segments to undergo a series of damages such as morphological 
changes, gene expression disorders, biochemical disorders, and metabolic changes [7], resulting in massive motor neuron death [38]. In the clinical treatment and animal model study of brachial plexus avulsion injury, replantation of avulsion nerve roots improves the survival rate of motor neurons after avulsion to a certain extent, and promotes the re-innervation and recovery of the motor function. However, replantation surgery does not completely restore the motor function of the upper limbs. The main reason is that before the target muscle atrophy of the upper limbs, the spinal motor neurons are unable to re-do the corresponding muscles because the axon growth rate is too slow. A large number of deaths of motor neurons after radiculopathy are considered to be important obstacles to the recovery of the motor function [12]. Therefore, the survival of motor neurons is considered to be an important prerequisite for the recovery of the motor function after brachial plexus injury [24]. In order to maintain the motor function of the upper limbs, after the injury, the motor neurons from the steady state to resist apoptosis and try to regenerate, but due to the adverse factors after the injury, the motor neurons eventually undergo irreversible death [11]. Therefore, further exploration of important signalling molecules and mechanisms to promote the survival and regeneration of motor neurons after injury will be of great significance for the prevention and treatment of brachial plexus avulsion.

(-)-epigallocatechin-3-gallate (EGCG), the predominant active polyphenol isolated from green tea, has been extensively investigated in a great deal of studies and considered to be a promising therapeutic agent for the treatment of diseases associated with chronic inflammation and oxidative damage $[1,30,39]$. EGCG inhibits the induction of iNOS transcription in lipopolysaccharide (LPS)-activated macrophages [26]. EGCG reduces airway inflammation $[15,35,41]$, free radical generation, and tumor necrosis factor $\alpha$ (TNF- $\alpha$ ) release [2] in animal models undergoing allergen-induced allergic diseases. EGCG targeting $\mathrm{HO}-1$ reduces contrast-induced kidney damage through anti-oxidative stress and anti-inflammatory pathways [17]. Previous studies have also shown that Erk1/2 signalling cascades exert a key role in the regulation of gene expression and prevention of apoptosis [29]. It is also reported that activation of the MAPK/Erk can promote the differentiation and survival of neurons [34,42].
Given protective roles of EGCG in multiple diseased systems, the neuroprotective effects of EGCG on motor neurons (MNs) were never proposed. Moreover, little is known about the underlying mechanism of EGCG on oxidative stress and inflammation in MNs in vitro. Therefore, the aim of this study is to investigate the effect of EGCG on brachial plexus root avulsion (BPRA), focusing on its anti-oxidative stress and neuroinflammation properties.

\section{Material and methods \\ Animals}

Four-week-old C57BL/6 mice obtained from the Guangdong Medical Laboratory Animal Centre (PR China) were maintained on a $12 \mathrm{~h}$ light/12 h dark cycle, and afforded food and water ad libitum. One male and 3 female mice were mated in each cage, and the offspring on PO were used for primary cultures of MNs. The Laboratory Animal Ethics Committee of the Xiangnan University approved all experimental protocols conducted on animals.

\section{Primary culture of motor neurons and treatments}

The primary culture of MNs was performed according to the previous studies with mirror modifications $[6,20,23,40]$. The spinal cord tissues from 0-day mice pups were dissected on the ice, and then digested with Hank's balanced salt solution (HBSS; 14175079, Thermo Fisher Scientific) containing $0.125 \%$ trypsin $(25200056$, Thermo Fisher Scientific) for $20 \mathrm{~min}$ at $37^{\circ} \mathrm{C}, 5 \% \mathrm{CO}_{2}$ atmosphere. After trituration, the purified dissociated cells were resuspended with DMEM/F-12 culture medium (SH30023, Solarbio Biotech Corp, Beijing, China) containing 10\% foetal bovine serum (FBS; F8245-100, Hangzhou Sijiqing bioengineering Materials Co., Ltd, Hangzhou, China) and 1\% Penicillin-Streptomycin (P/S; P1400, Solarbio Biotech Corp). The well-separated MNs were seeded in poly-D-lysine (PDL; D6790, Solarbio Biotech Corp)-coated 96-well plates at a concentration of $1 \times 10^{4}$ cells per well. After incubation for $4 \mathrm{~h}$ to allow the cell to adhere, the culture medium was replaced with Neurobasal-A medium (10888022, Thermo Fisher Scientific) supplemented with 2\% B-27 (17504044, Life Technologies) and $1 \% \mathrm{P} / \mathrm{S}$. The MNs were then incubated at $37^{\circ} \mathrm{C}$ in $5 \% \mathrm{CO}_{2}$ atmosphere overnight. 
To test the effect of EGCG on the MNs, cells were utilized with two different cell models: i) MNs were treated with Neurobasal-A medium containing $0,0.5,5,50$ or $500 \mu \mathrm{M}$ EGCG for $48 \mathrm{~h}$, followed by MTT assay; ii) MNs were exposed to $20 \mu \mathrm{M} \mathrm{H}_{2} \mathrm{O}_{2}$ for $2 \mathrm{~h}$ and then maintained in Neurobasal-A medium containing $50 \mu \mathrm{M}$ EGCG with or without the FIG4 antibody for $48 \mathrm{~h}$; iii) cells were treated with $50 \mu \mathrm{M}$ EGCG with or without the FIG4 antibody for $2 \mathrm{~h}$ prior to co-incubation with $1 \mu \mathrm{g} / \mathrm{ml}$ LPS for $24 \mathrm{~h}$. Finally, cell viability and ELISA assays were performed.

\section{Cell viability assay}

Cell viability assay was performed according to the previous studies with minor modifications [5,23]. The Cell Counting Kit (CCK-8; HY-K0301, MedChem Express, China) was used to evaluate cell viability. At the indicated time points, $10 \mu \mathrm{l}$ of CCK-8 solution was added to each well in 96-well culture plate, respectively, following a $2-\mathrm{h}$ incubation at $37^{\circ} \mathrm{C}$. And then, absorbance was measured in a multi-well plate reader (Tecan Infinite ${ }^{\circledR}$ M1000 Pro) at $490 \mathrm{~nm}$.

\section{Enzyme-linked immunosorbent assay}

Enzyme-linked immunosorbent assay (ELISA) kits manufactured by Hanhong Biochemical Company (Sino Biological, China) was used for measuring the FIG4 concentration in the primary cultured MNs. The $450 \mathrm{~nm}$ absorbance was measured by an ELISA reader (Tecan Infinite ${ }^{\circledR}$ M1000 Pro).

\section{Brachial plexus root avulsion and re-implantation model and grouping}

The surgical procedures were performed according to the previous studies with minor modifications [5,14,37]. Mice were anesthetized by avertin (T48402, Sigma-Aldrich) at a concentration of $13 \mu \mathrm{l} / \mathrm{g}$ and placed prone on a surgical table. After retracting the paravertebral muscles, the $\mathrm{C} 5-7 \mathrm{seg}$ ments of the spinal cord were easily identified. After right hemi-laminectomies at C4 to $\mathrm{C} 6$ and opening the dura, the C5-7 dorsal and ventral roots at the right side were avulsed. To build the re-implantation models, the C 6 ventral root was immediately re-implanted to the exact detachment point (on the pial surface) after the avulsion [4]. After muscles and skin were sutured in layers, all animals were recovered from anaesthesia and returned to their cages.
To investigate the neuroprotective role of EGCG after BPRA, 10 mice per group with re-implantation were randomly divided to 3 groups: (A) BPRA, (B) BPRA + EGCG and (C) BPRA + EGCG + FIG4 antibody. The treatment groups were intraperitoneally injected with $100 \mu$ l of phosphate buffer saline (PBS) or EGCG (the final concentration diluted in blood is $50 \mu \mathrm{M}$ ) with or without the FIG4 antibody once daily for 7 days after the surgery. The mice without BPRA or EGCG treatment were used as Sham control ( $n=10$ /group). No animals died during the 12-week survival time, after which the animals were sacrificed.

\section{Behaviour tests}

The grooming test, grasping test and cylinder test were performed according to the previous studies with minor modifications [5,9,16,37,43].

The TGT was performed at 6 weeks and 12 weeks after the surgery to evaluate the recovery of the motor function of the injured forelimb. 1-3 $\mathrm{ml}$ of purified water was sprayed on mice snout, and the movements of the forepaw to clear the water were videotaped for more than 5 min each time. The test scores were graded based on the position of the right forepaw [3]: 0 - no movement; 1 - elbow flexion but cannot touch the snout; 2 - elbow flexion and can touch the snout; 3 - elbow flexion and can reach below the eye; 4 - elbow flexion and can reach the eye; and 5 - elbow flexion and can reach the ear or back of the ear.

Generally, the grasping test was performed using the grip strength meter (GSM Grip Strength Meter 47200, Italy). The tail of the mice was gently lifted until only the tested paw grasped a grid connected to an ordinary electronic balance. And then, the mice were lifted further by the tail with the paw firmly grasping the grid. At the moment the paw lost its grip, the value shown by the electric balance was recorded. 5 measurements per forepaw were performed and recorded. The time interval between each measurement was $5 \mathrm{~min}$. The highest value in grams (g) was selected for the grasping strength for each mouse.

Briefly, the mice were placed in the CylinderScan (CSI, Gene\&I, Beijing, China), the times for the left and right paws to touch the wall of the CylinderScan were recorded, respectively. And the total times for the left and right paws were 20 . 


\section{Tissue preparation and immunofluorescence staining}

Tissue preparation and immunofluorescence staining were performed according to the previous studies with minor modifications [5]. Mice $(n=5 /$ group) were anesthetized deeply with isoflurane at 12 weeks post-surgery and transcardially perfused through the left ventricle with PBS followed by $4 \%$ paraformaldehyde (PFA) in PBS. The C5-7 segments of spinal cords were dissected and harvested. After being post-fixed in 4\% PFA for $24 \mathrm{~h}$ at $4^{\circ} \mathrm{C}$, spinal cord tissues were transferred to PBS containing $15 \%, 30 \%$ sucrose at $4^{\circ} \mathrm{C}$, respectively, and each for $24 \mathrm{~h}$. The sinking tissues were cut into sections at the thickness of $15 \mu \mathrm{m}$ on a sliding microtome (CM 1950, Leica, Germany). The C5-7 segments of each mouse were allocated to 4 alternating series of 10 sections each. The slides were blocked with $10 \%$ normal donkey serum (NDS) in PBS at RT for $1 \mathrm{~h}$, following the incubation overnight at $4^{\circ} \mathrm{C}$ with the following primary antibody: goat anti-choline acetyltransferase (ChAT, $1: 500$, AB144p, Millipore). After rinsing with PBS, immunoreactivity was visualized by incubation with Alexa Fluor 546 fluorescent secondary antibody (1: 1000, Invitrogen). The sections mounted using ProLong ${ }^{\circledR}$ Gold Anti-fade reagent with 4',6-diamidino-2-phenylindole (P36935, Gibco; Thermo Fisher Scientific) were imaged under an AxioObserver A1 microscope (Carl Zeiss, Oberkochen, Germany) using AxioVision 4.6 software (Carl Zeiss).

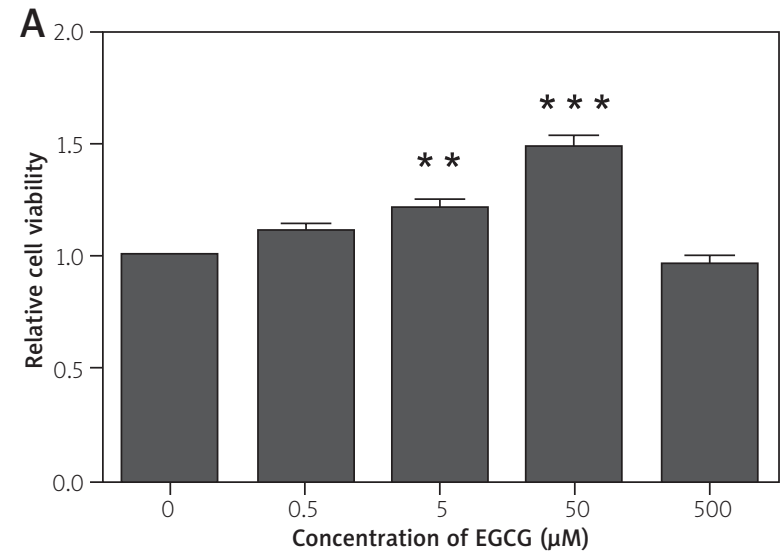

\section{Statistics}

All statistical analyses were performed by using GraphPad Prism 6 software. Data were reported as mean \pm standard deviation (SD) and analysed using ANOVA followed by the post-hoc Bonferroni test. $p<0.05$ was considered statistical significance.

\section{Results}

\section{EGCG increases the survival rate and the FIG4 expression in motor neurons in a dose-dependent manner}

To investigate the effect of EGCG on the primary cultured MNs, the cell viability assay and ELISA assay were performed after the MNs were treated with EGCG at various concentrations $(0,0.5,5,50,500 \mu \mathrm{M})$ for $48 \mathrm{~h}$.

We observed that the survival rate was increased in MNs after the treatment of EGCG in a dose-dependent manner, with a maximum effect observed at a concentration of $50 \mu \mathrm{M}$ (Fig. 1A). A similar pattern for the FIG4 levels in MNs was also observed (Fig. 1B).

\section{EGCG increases the survival rate$$
\text { of motor neurons under } \mathrm{H}_{2} \mathrm{O}_{2} \text { - }
$$$$
\text { induced oxidative stress and }
$$$$
\text { neuroinflammation via FIG4 }
$$

To investigate the effect of EGCG on the survival of MNs under oxidative stress and neuroinflammation, the cell viability assay was performed after the MNs were treated with $20 \mu \mathrm{M} \mathrm{H}_{2} \mathrm{O}_{2}$ for $2 \mathrm{~h}$ and

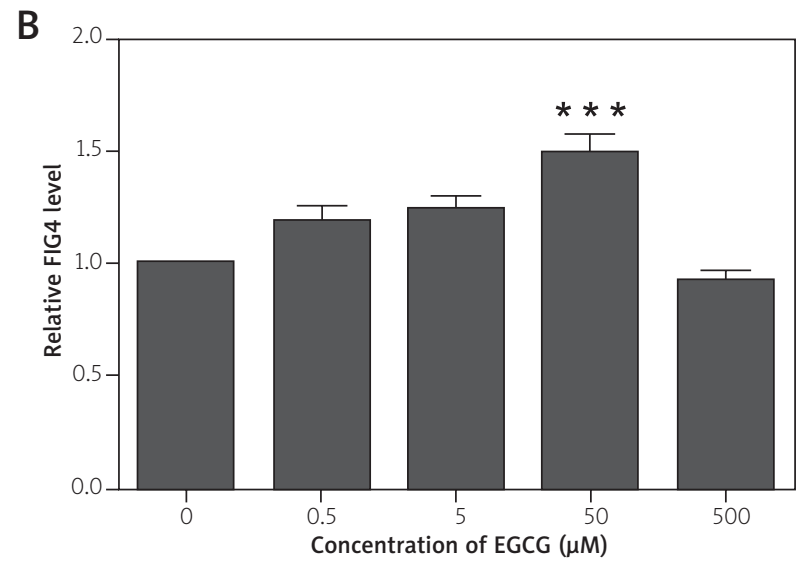

Fig. 1. Effect of EGCG on the cell viability and FIG4 expression in MNs in a dose-dependent manner in vitro. EGCG increased the peak levels of MNs survival and FIG4 expression at a concentration of $50 \mu \mathrm{M}(\mathbf{A}, \mathbf{B})$ $\left({ }^{* *} p<0.01,{ }^{* * *} p<0.0001, n=6\right)$. 

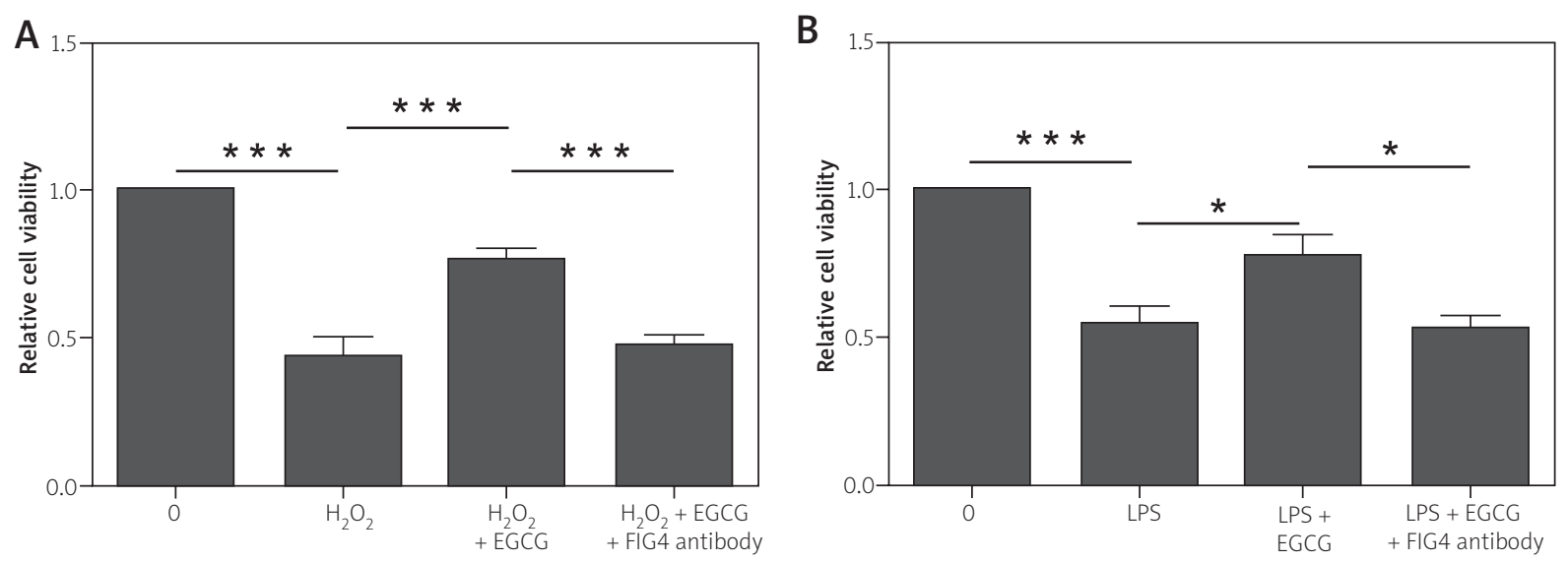

Fig. 2. Effect of EGCG on MNs survival under oxidative stress and neuroinflammation in vitro. A) EGCG protected against the cell death of $M N s$ induced by $\mathrm{H}_{2} \mathrm{O}_{2}$. B) EGCG protected against the cell death of MNs induced by LPS $\left({ }^{*} p<0.05,{ }^{* * *} p<0.0001, n=6\right)$.

following the treatment of $50 \mu \mathrm{M}$ EGCG for $48 \mathrm{~h}$ or pretreated with $50 \mu \mathrm{M}$ EGCG for $2 \mathrm{~h}$ and then co-cultured with $2 \mu \mathrm{g} / \mathrm{ml}$ LPS for $24 \mathrm{~h}$.

We found that treatment of $\mathrm{H}_{2} \mathrm{O}_{2}$ and LPS decreased the cell survival rates of MNs, whereas

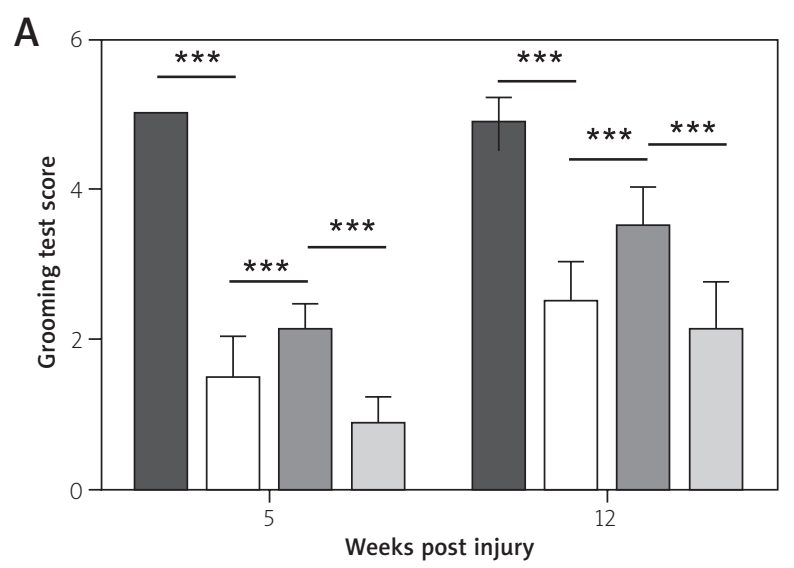

- Sham $\square$ BPRA $\square B P R A+E G C G \quad \square B P R A+E G C G+F I G 4$ antibody

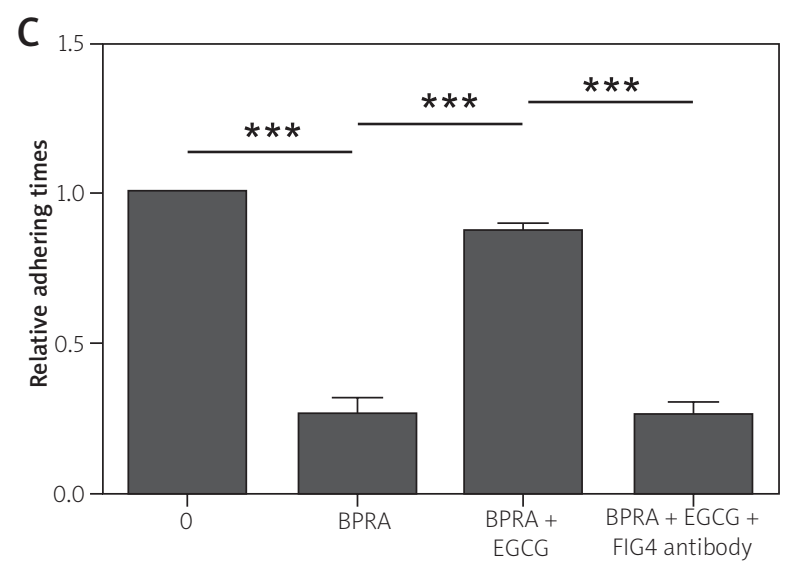

EGCG increased the cell survival of MNs induced by $\mathrm{H}_{2} \mathrm{O}_{2}$ and LPS; moreover, after treating the cells with the FIG4 antibody, EGCG cannot increase the cell survival of MNs induced by $\mathrm{H}_{2} \mathrm{O}_{2}$ and LPS (Fig. 2A, B).

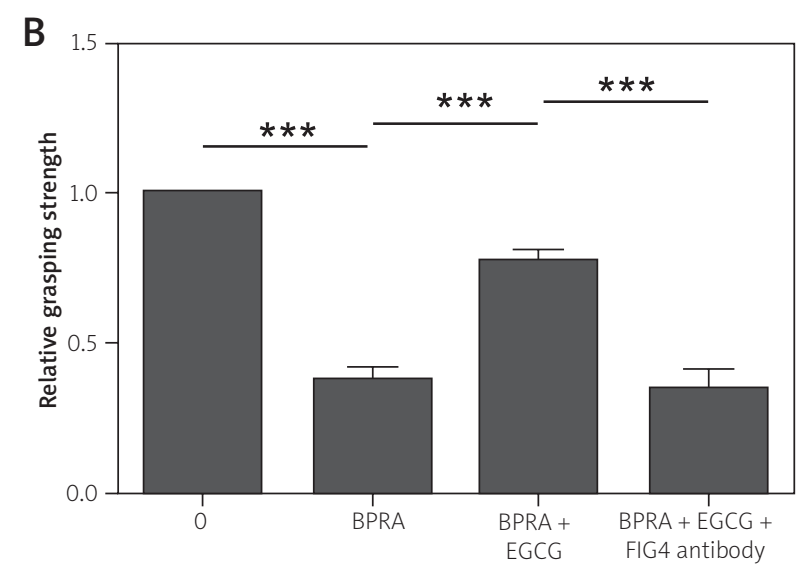

Fig. 3. Effect of EGCG on motor function recovery in mice following BPRA. A) Grooming test. (B) Grasping test, and (C) cylinder test were performed $\left({ }^{* * *} p<0.0001, n=10\right)$. 


\section{EGCG upregulates the FIG4 expression to promote the function recovery in mice following BPRA}

To investigate the effect of EGCG on the recovery of the motor function in mice after BPRA, the grooming test, grasping test and cylinder test were performed after the injury at 6 weeks or 12 weeks.

We found that the grooming test score was decreased in response to BPRA, whereas EGCG increased the grooming test score, moreover, after the treatment of the FIG4 antibody, EGCG cannot increase the grooming test score (Fig. 3A). The similar patterns for the grasping test and cylinder test in mice following BPRA were also observed (Fig. 3B,C).

\section{EGCG upregulates the FIG4 expression to increase the survival of motor neurons in the ventral horn in mice following BPRA}

To investigate the effect of EGCG on the survival of MNs in mice after BPRA, immunofluorescence staining was performed after injury at 12 weeks.
We found that the cell survival rates of MNs were decreased in response to BPRA, whereas EGCG increased the cell survival of MNs, moreover, after treating the cells with the FIG4 antibody, EGCG cannot increase the cell survival of MNs.

\section{Discussion}

In the present study, we observed that EGCG increases the survival rate of MNs after BPRA. Moreover, it also modulates FIG expression to be against the oxidative stress and neuroinflammation. This may suggest that EGCG may modulate FIG4 to promote the motor function recovery in mice after BPRA.

Several experimental studies have shown that EGCG can provide neuroprotection against brain, spinal cord injury and sciatic nerve injury $[28,36]$. These benefits are mainly due to free radical scavenging or the antioxidant, anti-inflammatory and anti-apoptotic properties of EGCG [32,33]. EGCG was verified to modulate the cell cycle and cell signalling [25] and be against liver injury via its anti-inflammatory and antioxidant effects [27]. In the present study, we indicate
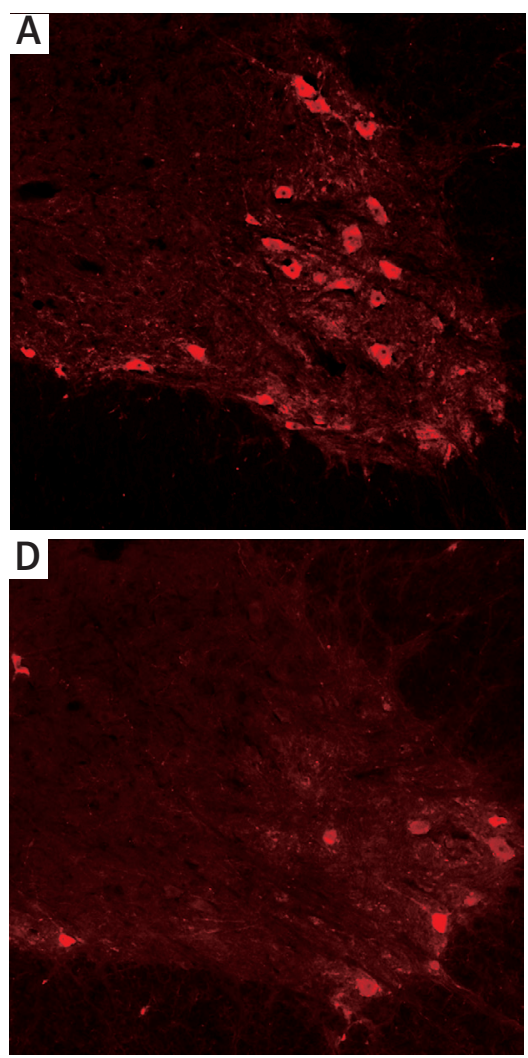
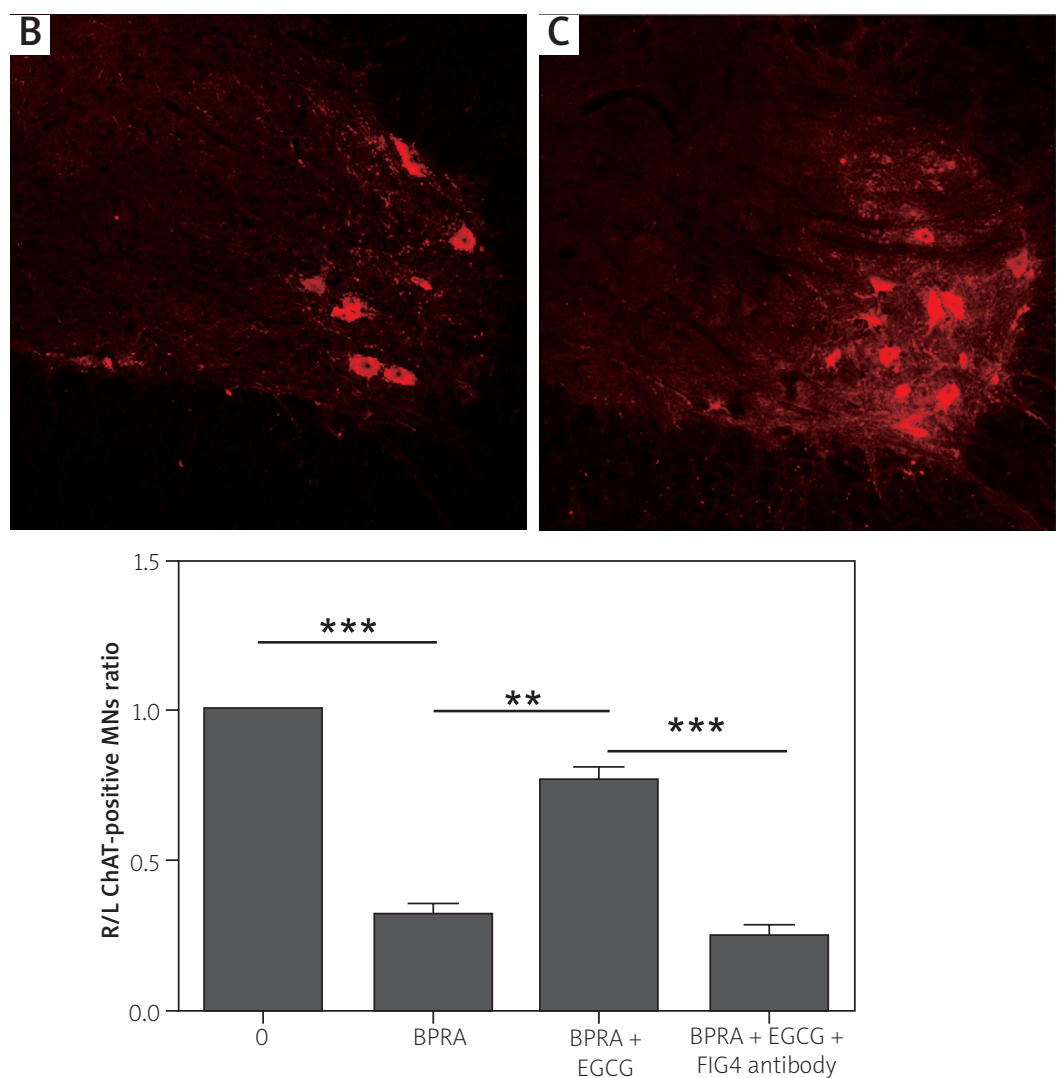

Fig. 4. Effect of EGCG on MN survival in the ventral horn in mice following BPRA. A-D) The number of MNs were decreased in response to EGCG treatment $\left({ }^{*} p<0.05,{ }^{* *} p<0.01,{ }^{* *} p<0.0001, n=5\right)$. 
that EGCG can promote the cell survival of MNs under oxidative stress and neuroinflammation.

FIG4, encoded by the FIG4 gene located on chromosome 6q21, is a phosphatase interacting with PIKfyve in mammalian cells or phosphatidylinositol-5-phosphate (PI5P) kinase Fab1 in yeast [18]. A protein complex formed by Fig4/PIKfyve and the kinase activators vac7 and 14 in yeast or arPIKfyve in mammalian cells regulates the yield of phophatidylinositol-3,5-diphosphate $(\mathrm{PI}, 5 \mathrm{P} 2)$. The loss of function of Fig4 may reduce the levels of PI3,5P2 [21,22]. In the nervous system Fig4/ PI3,5P2 signalling seems to be crucial for cell survival. Loss of FIG4 function in patients following Charcot-Marie-Tooth type 4J (CMT4J) may reduce approximately $71 \%$ of PI3,5P2 levels and be associated with severe neuronal degeneration $[8,31,44]$. In addition, vac14 (or arPIKfyve)-knockout mice show an approximately $50 \%$ decrease in the PI3,5P2 level and profound neuronal degeneration $[19,45]$.

Taken together, treatment with EGCG partially reverses the damage to MNs after BPRA via regulating FIG4. This may be helpful for the treatment of the neuronal injury, especially for the disorders related to MN survival.

Although the results look promising, our study still had some limitations: in this paper, we mainly concentrated on the survival of MNs according to the previous study [16], further studies are no doubt needed to be performed to evaluate the pathological changes of musculocutaneous nerve and biceps brachii muscles in response to the treatment of EGCG.

\section{Acknowledgements}

We thank the Research Foundation of Education Bureau of Hunan Province, China (No. 16B245) and the Hunan Province University Innovation Platform Open Fund Project, No. 16K082 and Scientific research projects of Chenzhou Science and Technology Bureau (NO.CZ-XNXY201509) for support.

\section{Disclosure}

The authors report no conflict of interest.

\section{References}

1. Alvarez E, Leiro J, Orallo F. Effect of (-)-epigallocatechin-3-gallate on respiratory burst of rat macrophages. Int Immunopharmacol 2002; 2: 849-855.

2. Bani D, Giannini L, Ciampa A, Masini E, Suzuki Y, Menegazzi M, Nistri S, Suzuki H. Epigallocatechin-3-gallate reduces allergen- induced asthma-like reaction in sensitized guinea pigs. J Pharmacol Exp Ther 2006; 317: 1002-1011.

3. Bertelli JA, Mira JC. Behavioral evaluating methods in the objective clinical assessment of motor function after experimental brachial plexus reconstruction in the rat. J Neurosci Methods 1993; 46: 203-208.

4. Chai H, Wu W, So KF, Prevette DM, Oppenheim RW. Oppenheim, long-term effects of a single dose of brain-derived neurotrophic factor on motoneuron survival following spinal root avulsion in the adult rat. Neurosci Lett 1999; 274: 147-150.

5. Chen S, Hou Y, Zhao Z, Luo Y, Lv S, Wang Q, Li J, He L, Zhou L, Wu W. Neuregulin-1 accelerates functional motor recovery by improving motoneuron survival after brachial plexus root avulsion in mice. Neuroscience 2019; 404: 510-518.

6. Chen SX, Hu CL, Liao YH, Zhao WJ. L1 modulates PKD1 phosphorylation in cerebellar granule neurons. Neurosci Lett 2015; 584: 331-336.

7. Cheng X, Fu R, Gao M, Liu S, Li YQ, Song FH, Bruce IC, Zhou LH, Wu W. Intrathecal application of short interfering RNA knocks down c-jun expression and augments spinal motoneuron death after root avulsion in adult rats. Neuroscience 2013; 241: 268-279.

8. Chow CY, Zhang Y, Dowling JJ, Jin N, Adamska M, Shiga K, Szigeti K, Shy ME, Li J, Zhang X, Lupski JR, Weisman LS, Meisler MH. Mutation of FIG4 causes neurodegeneration in the pale tremor mouse and patients with CMT4J. Nature 2007; 448: 68-72.

9. Denmark A, Tien D, Wong K, Chung A, Cachat J, Goodspeed J, Grimes C, Elegante M, Suciu C, Elkhayat S, Bartels B, Jackson A, Rosenberg M, Chung KM, Badani H, Kadri F, Roy S, Tan J, Gaikwad S, Stewart A, Zapolsky I, Gilder T, Kalueff AV. The effects of chronic social defeat stress on mouse self-grooming behavior and its patterning. Behav Brain Res 2010; 208: 553-559.

10. Dorsi MJ, Hsu W, Belzberg AJ. Epidemiology of brachial plexus injury in the pediatric multitrauma population in the United States. J Neuros-Pediatr 2010; 5: 573-577.

11. Eggers R, Tannemaat MR, De Winter F, Malessy MJ, Verhaagen J. Clinical and neurobiological advances in promoting regeneration of the ventral root avulsion lesion. Eur J Neurosci 2016; 43: 318-335.

12. Eggers R, Tannemaat MR, Ehlert EM, Verhaagen J. A spatio-temporal analysis of motoneuron survival, axonal regeneration and neurotrophic factor expression after lumbar ventral root avulsion and implantation. Exp Neurol 2010; 223: 207-220.

13. Faglioni W Jr., Siqueira MG, Martins RS, Heise CO, Foroni L. The epidemiology of adult traumatic brachial plexus lesions in a large metropolis. Acta Neurochir 2014; 156: 1025-1028.

14. Fang XY, Zhang WM, Zhang CF, Wong WM, Li W, Wu W, Lin JH. Lithium accelerates functional motor recovery by improving remyelination of regenerating axons following ventral root avulsion and reimplantation. Neuroscience 2016; 329: 213-225.

15. Fu M, Fu S, Ni S, Zou L, Liu Y, Hong T. Anti-inflammatory effect of epigallocatechin gallate in a mouse model of ovalbumin-induced allergic rhinitis. Int Immunopharmac 2017; 49: 102-108.

16. Fu R, Tang Y, Ling ZM, Li YQ, Cheng X, Song FH, Zhou LH, Wu $W$. Lithium enhances survival and regrowth of spinal motoneurons after ventral root avulsion. BMC Neurosci 2014; 15: 84.

17. Gao Z, Han Y, Hu Y, Wu X, Wang Y, Zhang X, Fu J, Zou X, Zhang J, Chen X, Jose PA, Lu X, Zeng C. Targeting HO-1 by epigallocatechin-3-gallate reduces contrast-induced renal injury via 
anti-oxidative stress and anti-inflammation pathways. PLoS One 2016; 11: e0149032.

18. Guo J, Ma YH, Yan Q, Wang L, Zeng YS, Wu JL, Li J. Fig4 expression in the rodent nervous system and its potential role in preventing abnormal lysosomal accumulation. J Neuropath Exp Neurol 2012; 71: 28-39.

19. Ikonomov OC, Sbrissa D, Delvecchio K, Xie Y, Jin JP, Rappolee D, Shisheva A. The phosphoinositide kinase PIKfyve is vital in early embryonic development: preimplantation lethality of PIKfyve-/- embryos but normality of PIKfyve+/- mice. J Bio Chem 2011; 286: 13404-13413.

20. Jiang Q, Chen S, Hu C, Huang P, Shen H, Zhao W. Neuregulin-1 (Nrg1) signaling has a preventive role and is altered in the frontal cortex under the pathological conditions of Alzheimer's disease. Mol Med Rep 2016; 14: 2614-2624.

21. Jin N, Chow CY, Liu L, Zolov SN, Bronson R, Davisson M, Petersen JL, Zhang Y, Park S, Duex JE, Goldowitz D, Meisler MH, Weisman LS. VAC14 nucleates a protein complex essential for the acute interconversion of $\mathrm{PI} 3 \mathrm{P}$ and $\mathrm{PI}(3,5) \mathrm{P}(2)$ in yeast and mouse, EMBO I 2008; 27: 3221-3234.

22. Lenk GM, Ferguson CJ, Chow CY, Jin N, Jones JM, Grant AE, Zolov SN, Winters JJ, Giger RJ, Dowling JJ, Weisman LS, Meisler MH. Pathogenic mechanism of the FIG4 mutation responsible for Charcot-Marie-Tooth disease CMT4J. PLoS Genet 2011; 7: e1002104.

23. Li J, Chen S, Zhao Z, Luo Y, Hou Y, Li H, He L, Zhou L, Wu W. Effect of VEGF on inflammatory regulation, neural survival, and functional improvement in rats following a complete spinal cord transection. Front Cell Neurosci 2017; 11: 381.

24. Li K, Cao RJ, Zhu XJ, Liu XY, Li LY, Cui SS. Erythropoietin attenuates the apoptosis of adult neurons after brachial plexus root avulsion by downregulating JNK phosphorylation and c-Jun expression and inhibiting c-PARP cleavage. J Mol Neurosci 2015; 56: 917-925.

25. Li Y, Zhao S, Zhang W, Zhao P, He B, Wu N, Han P. Epigallocatechin-3-O-gallate (EGCG) attenuates FFAs-induced peripheral insulin resistance through AMPK pathway and insulin signaling pathway in vivo. Diabetes Res Clin Pr 2011; 93: 205-214.

26. Lin YL, Lin JK. (-)-Epigallocatechin-3-gallate blocks the induction of nitric oxide synthase by down-regulating lipopolysaccharide-induced activity of transcription factor nuclear factor-kappaB. Mol Pharmacol 1997; 52: 465-472.

27. Liu D, Zhang X, Jiang L, Guo Y, Zheng C. Epigallocatechin-3-gallate (EGCG) attenuates concanavalin A-induced hepatic injury in mice. Acta Histochem 2014; 116: 654-662.

28. Machova Urdzikova L, Ruzicka J, Karova K, Kloudova A, Svobodova B, Amin A, Dubisova J, Schmidt M, Kubinova S, JhanwarUniyal M, Jendelova P. A green tea polyphenol epigallocatechin-3-gallate enhances neuroregeneration after spinal cord injury by altering levels of inflammatory cytokines. Neuropharmacology 2017; 126: 213-223.

29. McCubrey JA, Steelman LS, Abrams SL, Lee JT, Chang F, Bertrand FE, Navolanic PM, Terrian DM, Franklin RA, D’Assoro AB, Salisbury JL, Mazzarino MC, Stivala F, Libra M. Roles of the RAF/MEK/ ERK and PI3K/PTEN/AKT pathways in malignant transformation and drug resistance. Adv Enzyme Regul 2006; 46: 249-279.

30. Min SY, Yan M, Kim SB, Ravikumar S, Kwon SR, Vanarsa K, Kim HY, Davis LS, Mohan C. Green tea epigallocatechin-3-gallate suppresses autoimmune arthritis through indoleamine-2,3-dioxygenase expressing dendritic cells and the nuclear factor, erythroid 2-like 2 antioxidant pathway. J Inflam Lond 2015; 12: 53.
31. Nicholson G, Lenk GM, Reddel SW, Grant AE, Towne CF, Ferguson CJ, Simpson E, Scheuerle A, Yasick M, Hoffman S, Blouin R, Brandt C, Coppola G, Biesecker LG, Batish SD, Meisler MH. Distinctive genetic and clinical features of CMT4J: a severe neuropathy caused by mutations in the $\mathrm{PI}(3,5) \mathrm{P}(2)$ phosphatase FIG4. Brain 2011; 134: 1959-1971.

32. Ohishi T, Goto S, Monira P, Isemura M, Nakamura Y. Anti-inflammatory action of green tea. Anti Inflam Anti Allergy Agents Med Chemistry 2016; 15: 74-90.

33. Oliveira MR, Nabavi SF, Daglia M, Rastrelli L, Nabavi SM. Epigallocatechin gallate and mitochondria-A story of life and death. Pharmacol Res 2016; 104: 70-85.

34. Pearson G, Robinson F, Beers Gibson T, Xu BE, Karandikar M, Ber-man K, Cobb MH. Mitogen-activated protein (MAP) kinase pathways: regulation and physiological functions. Endocr Rev 2001; 22: 153-183.

35. Qin S, Alcorn JF, Craigo JK, Tjoeng C, Tarwater PM, Kolls JK, Reinhart TA. Epigallocatechin-3-gallate reduces airway inflammation in mice through binding to proinflammatory chemokines and inhibiting inflammatory cell recruitment. J Immunol 2011; 186: 3693-3700.

36. Renno WM, Benov L, Khan KM. Possible role of antioxidative capacity of (-)-epigallocatechin-3-gallate treatment in morphological and neurobehavioral recovery after sciatic nerve crush injury. J Neuros Spine 2017; 27: 593-613.

37. Ruven C, Badea SR, Wong WM, Wu W. Combination treatment with exogenous GDNF and fetal spinal cord cells results in better motoneuron survival and functional recovery after avulsion injury with delayed root reimplantation. J Neuropath Exp Neurol 2018; 77: 325-343.

38. Ruven C, Chan TK, Wu W. Spinal root avulsion: an excellent model for studying motoneuron degeneration and regeneration after severe axonal injury. Neural Regen Res 2014; 9: 117-118.

39. Shanmugam T, Selvaraj M, Poomalai S. Epigallocatechin gallate potentially abrogates fluoride induced lung oxidative stress, inflammation via Nrf2/Keap1 signaling pathway in rats: An in-vivo and in-silico study. Int Immunopharmac 2016; 39: 128-139.

40. Xu J, Hu C, Chen S, Shen H, Jiang Q, Huang P, Zhao W. Neuregulin-1 protects mouse cerebellum against oxidative stress and neuroinflammation. Brain Res 2017; 1670: 32-43.

41. Yang N, Zhang H, Cai X, Shang Y. Epigallocatechin-3-gallate inhibits inflammation and epithelialmesenchymal transition through the PI3K/AKT pathway via upregulation of PTEN in asthma. Int J Mol Med 2018; 41: 818-828.

42. Yuan Q, Su H, Chiu K, Wu W, Lin ZX. Contrasting neuropathology and functional recovery after spinal cord injury in developing and adult rats. Neurosci Bull 2013; 29: 509-516.

43. Zhang N, Luo Y, He L, Zhou L, Wu W. A self-assembly peptide nanofibrous scaffold reduces inflammatory response and promotes functional recovery in a mouse model of intracerebral hemorrhage. Nanomedicine 2016; 12: 1205-1217.

44. Zhang X, Chow CY, Sahenk Z, Shy ME, Meisler MH, Li J. Mutation of FIG4 causes a rapidly progressive, asymmetric neuronal degeneration. Brain 2008; 131: 1990-2001.

45. Zhang Y, Zolov SN, Chow CY, Slutsky SG, Richardson SC, Piper RC, Yang B, Nau JJ, Westrick RJ, Morrison SJ, Meisler MH, Weisman LS. Loss of Vac14, a regulator of the signaling lipid phosphatidylinositol 3,5-bisphosphate, results in neurodegeneration in mice. Proc Natl Acad Sci USA 2007; 104: 17518-17523. 